\title{
Orphan nuclear receptor COUP-TFII drives the myofibroblast metabolic shift leading to fibrosis
}

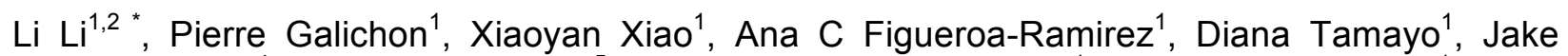
June-Koo Lee ${ }^{4}$, Marian Kalocsay, David Gonzalez-Sanchez ${ }^{1}$, Maria S Chancay ${ }^{1}$, Kyle McCracken ${ }^{1,2}$, Dario Lemos ${ }^{1,2}$, Nathan Lee ${ }^{1}$, Takaharu Ichimura ${ }^{1,2}$, Yutaro Mori ${ }^{1}$, M. Todd Valerius $^{1,2,3}$, Xiaoming Sun ${ }^{1}$, Elazer R Edelman ${ }^{2,6,7}$, Joseph V Bonventre ${ }^{1,2,3^{*}}$.

1 Renal Division, Brigham and Women's Hospital, Boston, Massachusetts, USA.

2 Department of Medicine, Harvard Medical School, Boston, Massachusetts, USA.

$3 \quad$ Harvard Stem Cell Institute, Cambridge, Massachusetts, USA.

4 Department of Biomedical Informatics, Harvard Medical School, Boston, MA, USA.

5 Department of Systems Biology, Laboratory of Systems Pharmacology, Harvard Medical School, Boston, MA, USA.

6 Cardiovascular Division, Brigham and Women's Hospital, Boston, MA, USA.

7 Institute for Medical Engineering and Science, Massachusetts Institute of Technology, Cambridge, MA, USA.

* Corresponding Authors

Address for Correspondence:

Li Li, MD

Division of Renal Medicine, Department of Medicine

Brigham and Women's Hospital, Harvard Medical School

Boston, MA, USA

Phone: 1-801-550-5529

Email: Ili29@bwh.harvard.edu

Joseph Bonventre, MD, PhD

Division of Renal Medicine, Department of Medicine

Brigham and Women's Hospital, Harvard Medical School

Boston, MA, USA

Phone: 1-617-525-5966

Email: jbonventre@bwh.harvard.edu 


\begin{abstract}
Recent studies demonstrated that metabolic disturbance, such as augmented glycolysis, contributes to fibrosis. The molecular regulation of this metabolic perturbation in fibrosis, however, has been elusive. COUP-TFII (also known as NR2F2) is an important regulator of glucose and lipid metabolism. Its contribution to organ fibrosis is undefined. Here, we found increased COUP-TFII expression in myofibroblasts in kidneys of patients with chronic kidney disease, fibrotic lungs of patients with idiopathic pulmonary fibrosis, fibrotic human kidney organoids, and fibrotic mouse kidneys after injury. Genetic ablation of COUP-TFII in mice resulted in attenuation of injury-induced kidney fibrosis. A non-biased proteomic study revealed the suppression of fatty acid oxidation and the enhancement of glycolysis pathways in COUP. TFII overexpressing fibroblasts. Overexpression of COUP-TFII in fibroblasts was sufficient to enhance glycolysis and increase alpha smooth muscle actin (aSMA) and collagen1 levels. Knockout of COUP-TFII decreased glycolysis and collagen1 levels in fibroblasts. Chip-qPCR assays revealed the binding of COUP-TFII on the promoter of PGC1a, a critical regulator of mitochondrial genesis and oxidative metabolism. Overexpression of COUP-TFII reduced the cellular level of PGC1a. In conclusion, COUP-TFII mediates fibrosis by serving as a key regulator of the shift in cellular metabolism of interstitial pericytes/fibroblasts from oxidative respiration to aerobic glycolysis. The fibrogenic response may share a common pathway in different organ injury and failure. Targeting COUP-TFII serves as a novel treatment approach for mitigating fibrosis in chronic kidney disease and potential other organ fibrosis.
\end{abstract}




\section{Introduction}

Kidney fibrosis is a pathologic hallmark of CKD, affecting approximately $10 \%$ of the world's adult population (Jha, Garcia-Garcia et al., 2013, Romagnani, Remuzzi et al., 2017). The progressive deposition and expansion of the fibrotic matrix in kidney parenchyma ultimately lead to kidney failure (Bonventre \& Yang, 2011, Rockey, Bell et al., 2015). A similar process was found in other fibrotic diseases, such as liver cirrhosis, idiopathic pulmonary fibrosis, and scleroderma (Wynn \& Ramalingam, 2012). The global burden of fibrosis is high, with an estimated prevalence rate at 1 in 4 people (Zhao, Kwan et al., 2019). Currently, there is no cure for fibrosis, highlighting the need for a new strategy and a better understanding of the molecular mechanisms underlying fibrosis. It is generally accepted that myofibroblasts are major cellular contributors to fibrotic disease (Bonventre \& Yang, 2011, Duffield, 2014, Duffield, Lupher et al., 2013, Tomasek, Gabbiani et al., 2002). Myofibroblasts express alpha smooth muscle actin $(\alpha S M A)$ and contract, migrate and produce excessive extracellular matrix (ECM) (Klingberg, Hinz et al., 2013). Comprehensive genetic fate mapping studies in rodents indicate that kidney resident Foxd1 expressing pericytes/perivascular fibroblasts/mesenchymal stem cell-like stromal cells are the main source of myofibroblasts after kidney injury (Falke, Gholizadeh et al., 2015, Humphreys, Lin et al., 2010, Humphreys, Valerius et al., 2008, Kobayashi, Mugford et al., 2014, Kramann, Schneider et al., 2015, Lin, Kisseleva et al., 2008, Picard, Baum et al., 2008).

Recently, an emerging body of evidence has demonstrated the link between metabolic dysregulation and fibrosis (Hou \& Syn, 2018, Lan, Geng et al., 2016, Xie, Tan et al., 2015, Zank, Bueno et al., 2018). Genome-wide transcriptome profiling revealed inflammation and metabolism as the top dysregulated pathways in fibrotic human kidneys (Kang, Ahn et al., 2015). A similar analysis of human skin fibrosis identified perturbations of fatty acid oxidation (FAO) and glycolysis pathways (Zhao et al., 2019). Inhibition of glycolysis or restoring FAO by genetic or pharmacological methods demonstrated promising results to mitigate fibrosis in various animal models (Ding, Jiang et al., 2017, Han, Wu et al., 2017, Kang et al., 2015, Tran, Zsengeller et al., 2016, Zhao et al., 2019). Despite these preliminary findings, the exact mechanisms that regulate metabolic dysregulation, especially in myofibroblast, remain largely unknown.

Chicken ovalbumin upstream promoter-transcription Factor II (COUP-TFII, also known as NR2F2) is an orphan member of the nuclear receptor family with unknown endogenous ligands (Pereira, Qiu et al., 1999). Since COUP-TFII has been reported to regulate metabolic functions (Ashraf, Sanchez et al., 2019, Li, Xie et al., 2009, Planchais, Boutant et al., 2015), we evaluated whether it played an important role in fibrosis. Downstream proteins under COUPTFII control tend to be involved in energy production, anabolic pathways, and cell cycle progression, all of which impact on cell proliferation (Chen, Qin et al., 2012, Planchais et al., 2015, Wu, Kao et al., 2015). Upregulation of COUP-TFII in numerous cancers, such as colon (Bao, Gu et al., 2014), pancreas (Polvani, Tarocchi et al., 2014), prostate (Qin, Wu et al., 2013), and renal cell carcinoma (Fang, Liu et al., 2020), further support the role of COUP-TFII in promoting cell proliferation. We hypothesized that it contributed to organ fibrosis via a regulatory role in the metabolism of the myofibroblast.

In this study, we demonstrate that COUP-TFII is a key regulator of shifting myofibroblast metabolism towards enhanced glycolysis with generation of pro-fibrotic mediators. In our studies increased COUP-TFII expression co-localized with aSMA expression in stromal cells of fibrotic human kidneys, lungs, kidney organoids, and fibrotic mouse kidneys after injury. Ablation of COUP-TFII in adult mice attenuated injury-induced kidney fibrosis. Our findings demonstrate a previously unrecognized role of COUP-TFII on regulating myofibroblast differentiation and highlight it as a relevant therapeutic target to prevent organ fibrosis after injury. 


\section{Results}

COUP-TFII expression is increased in myofibroblasts in human fibrotic diseases, and human kidney organoids

We interrogated previously published, human CKD microarray datasets and found a significant increase in COUP-TFII mRNA levels (1.9-fold) in renal biopsy tissues of 53 patients with CKD (GSE66494) (Fig. 1c) (Nakagawa, Nishihara et al., 2015). In another large cohort $(n=95)$ of microdissected human kidney samples from diabetic or hypertensive CKD subjects with pathology-defined fibrosis, microarray transcription profiling also revealed a significant upregulated COUP-TFII by analysis of total kidney mRNA levels (data not shown) (Kang et al., 2015). Results from these two independent cohorts of patients suggest an association between COUP-TFII mRNA expression and CKD in humans. We confirmed COUP-TFII protein expression and localization by immunofluorescent staining of normal and diseased human kidneys. In control 'healthy' kidney, which was obtained from the non-tumor portions of total nephrectomy samples in patients with renal cell carcinoma, we found little scattered COUP-TFII expression (Fig 1a). In the setting of kidney injury (patients with either acute thrombotic microangiopathy (TMA) or chronic diabetic nephropathy (DN)), however, the number of COUPTFII-positive cells was significantly increased (Fig 1a). The majority of these cells were localized in the interstitial region and co-localized with expanded aSMA-positive areas of fibrosis (Fig. 1a). Similar results were also identified in human fibrotic lung from patient with idiopathic pulmonary fibrosis (IPF) (Fig 1b).

Next, we examined COUP-TFII expression in human kidney organoids generated by directed differentiation of human pluripotent stem cells (Morizane, Lam et al., 2015). Human kidney organoids provide advantages of 3D nephron structures with multiple human kidney cell types and a rich stroma. Fibrosis of human kidney organoids was induced by incubation with IL$1 \beta$ for 96 hours as previous reported (Lemos, McMurdo et al., 2018). We then performed immunostaining of COUP-TFII on control and IL-1 $\beta$ treated organoids. As showed in Fig $1 \mathrm{~d}$, COUP-TFII expression significantly increased in IL-1 $\beta$ treated organoids compared to control. Most COUP-TFII positive cells were located in the interstitial region and co-localized with $\alpha$ SMA, as we observed in the kidneys of human subjects with CKD. Together, these results suggest an association of increased COUP-TFII expression with fibrosis in humans. The potential link to fibrosis prompted us to investigate the spatial and temporal expression, and mechanistic implications of COUP-TFII in non-injured and injured kidneys in adult mice.

\section{COUP-TFII protein is expressed in stromal cells in non-injured and injured mouse kidneys}

In healthy adult mouse kidneys, a few scattered COUP-TFII positive cells were present within the interstitium. The majority of COUP-TFII positive cells expressed PDGFR- $\beta$, a pericyte/fibroblast marker. COUP-TFII was not observed in endothelial cells marked by CD31 (Fig 2a).

To further assess the developmental origin of COUP-TFII+ cells, we crossed Foxd1-Cre driver mice (Humphreys et al., 2010) to tdTomato reporter mice (Madisen, Zwingman et al., 2010 ) to genetically label the Foxd1-derived stromal cells (Fig 2b). The majority of COUP-TFII protein expressing cells (green) overlapped with tdTomato-labeled Foxd1-derived stromal cells (red) (Fig 2c), demonstrating that COUP-TFII+ cells in non-injured mice derive from the Foxd1 population. Analysis of an available single-cell RNA sequencing database (http://humphreyslab.com/SingleCell/) confirmed that COUP-TFII RNA was most enriched in pericyte/fibroblast cells in injured kidneys, both in human (diabetic kidney) (Fig 2d top) and mouse (UUO day 14) (Fig 2d bottom). Thus the majority of COUP-TFII+ cells are kidney stromal cells, both in non-injured and injured kidneys. 


\section{COUP-TFIl expression is increased during the development of kidney fibrosis in various kidney injury models in mice}

COUP-TFII expression was significantly increased and co-localized in $\alpha S M A+$ cells within fibrotic regions in the injured kidney in two mouse kidney injury models: unilateral ureteral obstruction (UUO) and unilateral ischemia reperfusion injury (UIRI) (Fig 3a). To further characterize the spatial and temporal expression kinetics of COUP-TFII, we focused on the UUO model since it more reliably induces fibrosis in a short time frame. As shown in Fig 3b, COUP-TFII expression was upregulated as early as day 2 after injury, which is well before upregulation of $\alpha S M A$ and any histologic evidence of fibrosis was evident. We further demonstrated that the increased COUP-TFII expression is distinct from the inflammatory infiltrate that accompanies injury and fibrosis, as it does not overlap with markers of $T$ lymphocytes (CD3), neutrophils (LyG6), or macrophages (F4/80) (Fig 3c). Thus we conclude that COUP-TFII is upregulated specifically within the stromal compartment following kidney injury, and it precedes the expression of fibrotic markers, indicating a potential causative role in the pathophysiology of fibrosis.

Genetic lineage tracing analysis demonstrated that Gli1 marks perivascular mesenchymal stem cells-like cells, which are proposed to contribute to organ fibrosis (Kramann et al., 2015). We evaluated whether COUP-TFII+ cells and Gli1+ cells represent the same or closely related populations. We used the Gli1-CreERt2 line crossed with tdTomato reporter mice and induced genetic labeling 10 days prior to UUO injury (Fig 3d). Kidney tissues were collected at day 10 after UUO and stained for COUP-TFII expression. As expected, Gli1-derived cells expanded in number and acquired aSMA expressing 10 days after UUO (Fig 3e). Co-staining revealed that COUP-TFII expression was indeed found in nearly all of Gli1-tdTomato+ cells; however, there were also a large number of COUP-TFII+ cells that were distinct from the Gli1 lineage (Fig $3 e$ ). Although some of these cells could result from incomplete labeling with the inducible Gli1-Cre, there was also a noticeable difference in the spatial distribution between the COUP-TFII+/tdTomato- and COUP-TFII+/tdTomato+ populations. Interestingly, while COUPTFII+ cells were found throughout both the cortex and medulla, co-localizing with aSMA+ cells in both regions, the Gli1-tdTomato+ cells were largely restricted to the outer medullary region (Fig 3f). This is consistent with the previous observations (Kramann et al., 2015), that Gli1tdTomato+ cells represented only a small fraction of the total PDGFR $\beta+$ population. Gli1 cells were enriched in the outer medulla with much less expression in pericytes and perivascular fibroblasts of the cortex(Humphreys, 2018, Kramann et al., 2015). As fibrosis is present both in cortex and outer medulla, our data suggest that COUP-TFII functions more generally in regulating pericyte to myofibroblast differentiation, irrespective of the anatomic compartments.

\section{Ablation of COUP-TFII in adult mice attenuates injury-induced kidney fibrosis}

Since global knockout of COUP-TFII in mice confers embryonic arrest at E10 due to defective angiogenesis and cardiac development (Pereira et al., 1999), we used a tamoxifen-inducible Cre-loxP system to knockout COUP-TFII in adult mice. We generated COUP-TFII ${ }^{\text {flox } /+}$; Rosa26 $^{\text {CreERT2/+ }}(\mathrm{F} /+; \mathrm{Cre} /+)$ and COUP-TFII ${ }^{\text {flox/flox; }}$; Rosa26 ${ }^{\text {CreERT2/+ }}$ (F/F; Cre/+) mice. COUP-TFII expression was maintained in these mice without tamoxifen (TAM). Three injections of TAM activated Cre recombinase and generated COUP-TFII heterozygous (+/-) and homozygous (-/-) knockout mice. Ten days after TAM injection, mice were subjected to UUO and euthanized 7 days after UUO (Fig 4a). There was no identifiable phenotypic difference between WT and knockout mice without injury.

We first examined the efficacy of COUP-TFII ablation. A LacZ knock-in allele was inserted into the genomic COUP-TFII locus after the second LoxP site (Takamoto, You et al., 2005). Treatment with Cre recombinase resulted in COUP-TFII deletion and the expression of the LacZ reporter. Using this LacZ reporter, we were able to trace the COUP-TFII lineage 
following deletion. As shown in Fig $4 \mathrm{~b}$, expression of $\beta$-galactosidase ( $\beta-\mathrm{Gal}$ ) increased after TAM injection in UUO kidneys of heterozygous mice with Cre allele, but not in the control mice with flox allele but without Cre allele (WT), indicating the specificity of $\beta$-Gal staining in the CreLoxP system. Expression of COUP-TFII decreased after TAM injection in heterozygous (+/-) mice. Ten days after TAM injection, $\beta-G a l$ expression co-localized with COUP-TFII expression in both contralateral and UUO kidneys, consistent with the deletion of one allele of COUP-TFII (Fig 4c). Both $\beta$-Gal expression and COUP-TFII expression increased in UUO kidney compared to contralateral non-injured kidney (Fig 4c). $\beta$-Gal staining demonstrated that this population remained stable following TAM injection, indicating that COUP-TFIl deletion does not impact the survival or proliferation of these cells at baseline.

Next we examined the effect of COUP-TFII ablation on injury-induced kidney fibrosis (UUO model) using knockout (KO, -/-) mice. As shown in Fig 4d, COUP-TFIl positive cells decreased significantly in a KO (-/-) mice after UUO compared with WT kidneys, indicating successful knockout of COUP-TFII. Associated with much less COUP-TFII expression, $\alpha$ SMA and collagen1 expression was significantly decreased in KO compared to WT at 7 days after UUO. Histological evaluation demonstrated less kidney fibrosis in KO compared to WT by Masson Trichrome (Fig 4d). These data demonstrated that ablation of COUP-TFII in adult mice attenuates injury-induced kidney fibrosis.

\section{COUP-TFII regulates myofibroblast differentiation in vitro through metabolic reprogramming}

In order to dissect the mechanistic role of COUP-TFII in myofibroblast differentiation, we generated COUP-TFII loss- and gain-of-function cell lines using CRISPR-Cas9 (clustered regularly interspaced short palindromic repeats (CRISPR)-CRISPR associated protein 9 (Cas9)) and an inducible lentiviral construct, respectively, in the pericyte-like cell line $\mathrm{C} 3 \mathrm{H} / 10 \mathrm{~T} 1 / 2$. $\mathrm{C} 3 \mathrm{H} / 10 \mathrm{~T} 1 / 2$ is a mouse mesenchymal cell that has been used in studies related to pericyte biology and cell fate determination in vitro [45-47]. Naïve C3H/10T1/2 cells (WT) showed basal expression of COUP-TFII, which we were able to successfully modulate using the knockout (KO) or overexpression (OE) systems (Fig 5a). Neither KO nor OE affected cell viability (Fig $5 \mathrm{~b}$ ), although a decreased proliferation rate in $\mathrm{KO}$ cells and increased proliferation rate in OE cells were observed compared to naïve (WT) cells (Fig 5c). Treatment of C3H/10T1/2 cells with TGF $\beta 1$ induces myofibroblast differentiation with up-regulation of $\alpha \mathrm{SMA}$, and this effect was preserved in KO and OE cells (Fig 5d). Interestingly, OE cells, in the absence of TGF $\beta 1$ stimulation, displayed an elongated fibroblast shape, similar to WT cells treated with TGF $\beta 1$ (Fig 5d). More importantly, there was increased expression of aSMA and collagen1 in COUP-TFII OE cells even in the absence of TGF 1 stimulation (Fig $5 \mathrm{~d}$-e), while their expression was significantly diminished in the KO cells. Collectively, these data further support a profibrotic function for COUP-TFII in pericyte/fibroblast-like cells.

To further interrogate the molecular mechanisms by which COUP-TFII regulates myofibroblast differentiation, we evaluated the proteome of WT and OE cells that were differentiated with TGF $\beta 1$. This non-biased approach unequivocally implicated cellular metabolic pathways as predominant targets of COUP-TFII. Gene set enrichment analysis (GSEA) highlighted that the most significantly down-regulated proteins in COUP-TFII-OE cells were enriched in mitochondrial electron transport chain and fatty acid oxidation (FAO) pathways (Fig $6 a)$, which are involved in oxidative metabolism. Conversely, the top up-regulated pathways in COUP-TFII-OE cells were associated with both extracellular matrix, including collagen fiber organization, cadherin binding and actin cytoskeleton, and glycolysis pathways (Fig 6a \& b). Taken together, these data support a model in which COUP-TFII shifts cell metabolism from FAO toward glycolysis, which may lead to increased extracellular matrix production and fibrosis. Indeed we found that COUP-TFII overexpression was sufficient to significantly increase 
expression of key components of the glycolytic pathway, including Glut1, Hexokinase2 (HK2) and Lactate dehydrogenase A (LDHA) (Fig 6c). To directly test the link between glycolytic metabolism and myofibroblast differentiation, we inhibited glycolysis with 2-deoxyglucose (2DG), a well-defined HK2 inhibitor. In the COUP-TFII OE cells, 2-DG attenuated the TGF $\beta 1$ induced expression of aSMA and collagen 1 at both the mRNA and protein level (Fig 6d). These data suggest that COUP-TFII enhances TGF $\beta 1$-induced glycolysis during myofibroblast differentiation, and that the switch to glycolysis is essential for this process.

Having demonstrated that COUP-TFII-OE cells adopt enhanced glycolysis and profibrotic phenotype in response to TGF $\beta 1$, we further examined this model in the KO cell line. Using the Seahorse X24 extracellular flux analyzer, we found that the COUP-TFII KO cells exhibited significant reduction in both the glucose-induced glycolytic preference and the oligomycin-induced total glycolytic capacity when compared to WT cells, measured by extracellular acidification rate (ECAR) (Fig 6e). Interestingly, KO cells has a lower oxygen consumption rate (OCR), a measure of mitochondrial respiratory activity, in response to TGF $\beta 1$ compared with WT cells (Fig 6e). This is consistent with the slower growth of KO cells compared to WT cells. To confirm modifications in glycolysis induced by COUP-TFII perturbation, we directly measured the levels of lactate in the culture medium. TGF $\beta 1$ significantly increased lactate production in WT cells, which was blunted by COUP-TFII KO. Overexpression of COUP-TFII alone (without TGF $\beta 1$ ) significantly increased lactate levels (Fig 6f), indicating augmented glycolysis in these cells. Furthermore, overexpressing COUP-TFII resulted in decreased transcription of PGC1 $\alpha$ and knockout of COUP-TFII increased PGC1a mRNA in vitro (Fig 6g). This is consistent with our Chip-qPCR results, in which PGC1a is a target of COUP-TFII (Fig 6h), Altogether, these data demonstrated that COUP-TFII augmented glycolysis and promoted myofibroblast differentiation, and collagen1 production after injury (Fig $6 i)$.

\section{Discussion}

Pericyte/perivascular cells are important contributors to kidney fibrosis after injury (Humphreys et al., 2010, Lin et al., 2008). These cells are the main source of myofibroblasts, the effector cells for fibrosis (Duffield, 2014, Humphreys, 2018). Blocking the differentiation of pericyte/perivascular cells to myofibroblasts is an attractive strategy to reduce fibrosis. TGF $\beta$, a master regulator of myofibroblast differentiation, has been extensively studied as a therapeutic target (Akhurst \& Hata, 2012, Meng, Huang et al., 2012, Rangarajan, Kurundkar et al., 2016). Direct inhibition of TGF $\beta$, however, has led to more toxicity than benefit (Li, Wan et al., 2006, Principe, Doll et al., 2014). Therefore, alternative molecular approaches to the regulation of myofibroblast differentiation during fibrosis development are desirable for anti-fibrotic drug development.

We demonstrated that COUP-TFII is markedly increased in human kidneys and lungs with fibrosis or cells in human kidney organoids activated with IL-1 $\beta$ to enhance stromal fibrosis. COUP-TFII is expressed in pericytes/perivascular cells in adult non-injured kidney, and colocalizes with aSMA expression during fibrosis formation after kidney injury. Another protein proposed to be important in the conversion of pericytes/perivascular cells to myofibroblasts is Gli1 (Kramann et al., 2015). In comparison with Gli1, COUP-TFII is seen in more $\alpha$ SMA+ cells, including many that are not Gli1-tdTomato+, especially in the kidney cortex. Increased COUPTFII expression after injury has important functional significance for the myofibroblast population. Using a tamoxifen-induced Cre-loxP system, we deleted COUP-TFII in adult mice and evaluated its role in injury-induced kidney fibrosis. Genetic depletion of COUP-TFII reduced $\alpha S M A$ positive cells and attenuated kidney fibrosis after injury. Our results demonstrate that COUP-TFII plays a pivotal role in myofibroblast differentiation and kidney fibrosis formation. We 
also demonstrated increased COUP-TFII expression in myofibroblasts in fibrotic lungs of patients with idiopathic lung fibrosis (IPF). These findings implicate the general role of COUPTFII in myofibroblast during fibrosis formation.

Giving the important role of COUP-TFII on glucose and lipid metabolism (Ashraf et al., 2019), we hypothesized that COUP-TFII might regulate myofibroblast differentiation through metabolism reprogramming. Our proteomic data revealed that COUP-TFII promotes the expression of proteins enriched in metabolic process critical for myofibroblasts, in particular, suppression of FAO and enhancement of glycolysis. Our data link COUP-TFII to the metabolic switch from oxidative metabolism to aerobic glycolysis leading to a proliferative and pro-fibrotic function of myofibroblast. Overexpression of COUP-TFII alone (without TGF $\beta 1$ treatment) is sufficient to increase glycolysis and collagen1 expression. Knockout of COUP-TFIl dampened TGF $\beta 1$-induced glycolysis and decreased $\alpha$ SMA and collagen 1 expression. The phenotypic resemblance between COUP-TFII overexpressed alone cells and WT cells treated with TGF 1 suggests that COUP-TFII is essential to establish the myofibroblast phenotype through augmented glycolysis. High glycolytic flux is important for the self-renewal of progenitor cells (Liu, Edgington-Giordano et al., 2017). Interestingly, COUP-TFIl expression is abundant in the mesenchymal compartment of the developing organs during embryonic organogenesis but declines significantly right after birth(Pereira, Qu et al., 1995). It is tempting to suggest that COUP-TFII might be important to maintain the de-differentiation status of cells through augmented glycolysis during development.

Besides supporting energy needs in a hypoxic environment, glycolysis also provides macromolecules required for cell proliferation and migration (Xie et al., 2015). In this way, the reprogramming of cell metabolism ensures sufficient building blocks for biosynthesis and facilitates survival of myofibroblasts in a harsh hypoxic and nutrient-deprived microenvironment. Furthermore, glycolysis is clearly linked to ECM production (Ding et al., 2017, Zhao et al., 2019). Collagen1, the predominant structural protein found in kidney fibrosis, is synthesized through multiple steps, including hydroxylation of amino acids, disulfide bonding and glycosylation (Basak, Vega-Montoto et al., 2016). The major amino acid components of collagen are glycine, proline, and lysine. Glycine is mainly produced from glycolysis (de Paz-Lugo, Lupianez et al., 2018). In addition, collagen hydroxylation and glycosylation are depended on glycolysis (Im, Freshwater et al., 1976). Our data, along with studies from others (Ding et al., 2017, Xie et al., 2015), demonstrate that 2-DG (an hexokinase inhibitor) drastically decreases collagen 1 production in myofibroblasts in vitro. There is emerging evidence showing that augmented glycolysis in cancer stromal cells (also call cancer associated fibroblasts) support cancer progression through secreting lactate and other glycolytic intermediates (Avagliano, Granato et al., 2018). In addition, a decrease in microenviromental $\mathrm{pH}$ from lactic acid accumulation has been associated with increased TGF $\beta$ activity (Kottmann, Kulkarni et al., 2012). Therefore, targeting glycolysis in myofibroblasts would be expected to not only inhibit the activation of stromal cells, but also to modify the microenvironment of fibrosis foci. We report that this molecular regulation of metabolic reprogramming is likely facilitated by the function of COUPTFII to decrease PGC1 $\alpha$ transcription given that COUP-TFII binds to the PGC1 $\alpha$ promoter.

Our study shows that with fibrosis COUP-TFII is expressed primarily in myofibroblasts. Since COUP-TFII is expressed primarily in myofibroblasts, targeting it may be selectively effective to suppress myofibroblast differentiation and function without affecting epithelial or endothelial cells. There are no overt phenotypes in COUP-TFII KO mice at the adult stage further supporting the potential safety profile of COUP-TFII inhibition. Given that COUP-TFII expression is upregulated in fibrotic human kidney organoids, these ex vivo human cell systems can be used to test potential inhibitors.

In conclusion, our study provides compelling evidence that COUP-TFII regulates myofibroblast differentiation and profibrotic function through augmented glycolysis after injury. Reducing COUP-TFII is effective in diminishing myofibroblast differentiation and limiting fibrosis. 
The fibrogenic response may share a common pathway in different organ injury and failure. Targeting COUP-TFIl serves as a novel treatment approach for mitigating fibrosis in chronic kidney disease and potential other organ fibrosis.

\section{Methods and Material}

\section{Human kidney biopsy sample preparation and immunostaining}

Normal kidney samples used for immunostaining studies were obtained from surgical sections from patients undergoing nephrectomy due to renal cell carcinoma (RCC) under institutional review board-approved protocols. Injured kidney samples were obtained form kidney biopsy samples from patients with thrombotic microangiopathy (TMA) and diabetic nephropathy (DN).

\section{Human kidney organoids generation and IL-1 $\beta$ stimulation}

Kidney organoids were derived from $\mathrm{H} 9$ human embryonic stem cells as previously described (Morizane et al., 2015). Briefly, H9 cells were cultured in StemFit (Ajinomoto) supplemented with $10 \mathrm{ng} / \mathrm{ml}$ FGF2 (Peprotech). Differentiation was started with $8 \mathrm{vM} \mathrm{CHIR} \mathrm{(TOCRIS)} \mathrm{for} 4$ days, followed sequentially by Activin for 3 days and FGF9 for 1-2 days. Subsequently cells were dissociated with Accutase (Stem Cell Technologies) and plated into U-shaped bottoms 96-well plates (Corning) at 100,000 cells per well in a medium supplemented with $3 \mu \mathrm{M} \mathrm{CHIR}$ and 10 $\mathrm{ng} / \mathrm{ml} \mathrm{FGF9}$. Two days later the medium was changed to one supplemented with only $10 \mathrm{ng} / \mathrm{ml}$ FGF9. After 3-4 days, the medium was changed to basal medium without additional growth factors. At day 51, matured organoids were treated with IL-1 $10 \mathrm{ng} / \mathrm{ml}$ (Sigma) for 96 hours. Organoids were collected and fixed in 4\% PFA for 30 minutes followed by $20 \%$ sucrose overnight. Cryosections $(7 \mu \mathrm{m})$ were incubated with antibodies for immunofluorescence. Images were captured by confocal microscopy using a Nikon C1 microscope running EZ-C1 software.

\section{Mouse strain and animal experiments}

All mouse experiments were performed under the animal use protocol approved by the Institutional Animal Care and Use Committee of the Brigham and Women's hospital. Gli1CreER $^{\text {t2 }}$ (JAX\# 007913), Rosa26tdTomato (JAX\# 007909), Foxd1-GFP-Cre (Jax\# 012463), Rosa26-CreER ${ }^{\mathrm{t} 2}$ (JAX\# 008463) were purchased from Jackson Laboratories (Bar harbor, ME). COUP-TFII flox/+ mice were purchased from Mutant Mouse Resource \& Research Centers (MMRRC) (B6;129S7-Nr2f2tm2Tsa/Mmmh, Cat\# 032805-MU). The COUP-TFII flox/+ mouse strain was maintained in a mixed genetic background (129/Sv x C57BL/6) and received standard rodent chow. To induce COUP-TFII deletion in the adult, 8-12 weeks old mice were intraperitoneally injected with 3 doses $0.1 \mathrm{mg} / \mathrm{g}$ body weight tamoxifen in corn oil $/ 3 \%$ ethanol (Sigma) every other day starting 14 days before surgery.

Murine kidney fibrosis models were performed as previously described (Yang, Besschetnova et al., 2010). Briefly, mice were anesthetized with pentobarbital sodium (60 $\mathrm{mg} / \mathrm{kg}$ body weight, intraperitoneally). For the unilateral ureteral obstruction (UUO) surgery, a flank incision was made and the left ureter was tied off at the level of the lower pole with two 4.0 silk ties. For the unilateral ischemia reperfusion injury (IRI), the left kidney was exposed through a flank incision and subjected to ischemia by clamping the renal pedicle with non-traumatic microaneurysm clamps (Roboz, Rockville, MD) for 30 minutes. Reperfusion was confirmed by visual color change. Body temperatures were controlled at $36.5^{\circ} \mathrm{C}-37.5^{\circ} \mathrm{C}$ throughout the procedure. One milliliter of warm (body temperature) saline was instilled in the retroperitoneum after surgery for volume supplement. Buprenorphine was used for pain control $(0.1 \mathrm{mg} / \mathrm{kg}$ body weight, intraperitoneally). Mice were sacrificed at day 2, 5 or 10 after UUO and day 14 after unilateral IRI.

\section{Histology and immunofluorescence staining}


Mice were anesthetized with isofluorane (Baxter) and subsequently perfused via the left ventricle with $4^{\circ} \mathrm{C}$ PBS for 1 minute. Kidneys from adult mice were fixed with $4 \%$ paraformaldehyde (PFA), dehydrated and embedded in paraffin. Hematoxylin/eosin, PAS and Masson's trichrome staining were performed using standard protocols (Yang et al., 2010).

Immunofluorescence staining of mouse kidneys was performed on paraffin sections as previously described (Yang et al., 2010). Briefly, the tissue sections were deparaffinized, followed by antigen retrieval and rehydration. Then tissue antigens were labeled with primary antibodies to COUP-TFII (Abcam, diluted 1: 200), aSMA (Sigma, 1:400), collagen1 (EMD Millipore, 1:400), CD31 (Abcam 1: 200), PDGF receptor beta (PDGFR $\beta$, Abcam 1:400), or $\beta$ galactosidase (Abcam 1:100), followed by FITC or Cy3-labeled secondary antibodies (Jackson ImmunoResearch). Some immunostaining was performed on frozen sections. Cryosections (7 $\mu \mathrm{m})$ were fixed in $4 \%$ PFA for 2 hours, and then, washed in $30 \%$ sucrose solution overnight. Primary antibodies used in cryosections recognized the following proteins: CD3 (eBioscience 1:100), LyG6, F4/80 (ThermoFisher), Images were captured by a confocal (Nikon C1) or standard fluorescent microscope (Nikon TE 1000).

\section{Cell Culture and treatment}

The C3H10T1/2 (American Type Culture Collection) were cultured in DMEM medium supplemented with $10 \%$ FCS until the cells were $80 \%$ confluent. Cell morphology was examined and captured using live cell imaging (Nikon). For cytokine induction of myofibroblast differentiation, subconfluent cells were incubated in DMEM medium containing $1 \%$ BSA overnight and treated with TGF $\beta 1$ (10ng/ml) (R\&D).

\section{CRISPR/Cas9 knockout}

COUP-TFIl guide RNAs were created using a guide design web tool (http://crispr.mit.edu): COUP-TFII guide 1, TATATCCGGACAGGTACGAG; COUP-TFII guide 2, GAGGGGGTCCCCGTTGGTCA. sgRNA oligos were cloned into pSpCas9(BB)-2A-GFP (Addgene, 48138). The protocol was performed according to published methods (Ran, Hsu et al., 2013). C3H10T1/2 cells $\left(5 \times 10^{4}\right)$ were transfected with lipofectamine 3000 . Cells were grown for $48 \mathrm{~h}$ and then sorted for GFP and positive cells seeded as single cells in 96-well plates. Cells were then returned to the incubator and cultures were allowed to expand for 2-3 weeks.

\section{Tet-Inducible COUP-TFIl expression}

To generate a lentiviral transfer plasmid for inducible gain-of-function experiments, we used high-fidelity PCR (iProof, BioRad) to amplify full-length mouse COUP-TFII cDNA, flanked with attB1 and attB2 sites on 5' and 3' ends respectively, from a cDNA library from adult mouse kidney. The resulting PCR band was purified from a $0.8 \%$ agarose gel using QIAquick Gel Extraction kit (Qiagen). The purified PCR product was then cloned into pDONR221 (Thermo Fisher Scientific) using BP Clonase II (Invitrogen) according to manufacturer's instructions. We then shuttled the COUP-TFII cDNA into the destination vector plnducer20 (a gift from Stephen Elledge, Addgene \#44010) using LR Clonase II (Invitrogen). All cloning steps were verified using Sanger sequencing (Genewiz, Inc.). C3H10T1/2 cells were infected with lentivirus in the presence of $10 \mu \mathrm{g} / \mathrm{ml}$ polybrene (Sigma). Infected cells were selected with puromycin (Sigma).

\section{Cell proliferation assay}

Cells were seeded on a 96-well plate at a concentration of $1 \times 10^{4}$ per well. Three parallel wells of cells were studied for each group. After incubation for 1 day, 2 days, or 3 days, $20 \mu \mathrm{L}$ MTT ((3-(4,5-Dimethyl-2-thiazolyl)-2,5-diphenyltetrazolium Bromide), promega) was added. After 2 hours of MTT exposure, cells were washed and subjected for colorimetric measurement. The 
OD values were obtained by a microplate reader (SpectraMax M5, Molecular Devices) at $570 \mathrm{~nm}$.

\section{Quantitative real-time PCR (qRT-PCR)}

At indicated times, total RNA was extracted using TRIzol (Sigma) as described (Yang et al., 2010). Subsequently, $2 \mu \mathrm{g}$ of total RNAs was reverse-transcribed to cDNA with random primers using reverse transcriptase (Invitrogen). A 1: 5 dilution of cDNA was then amplified by real-time qPCR in a CFX96 real-time system (Biorad) using SYBR green. Relative gene expression was calculated by the $\triangle \Delta \mathrm{Ct}$ method, and final results were expressed as the fold difference relative to control conditions in gene expression normalized to Ribosomal Protein L32 (RPL32). Primers for individual gene expression were listed in Table 1.

\section{ChIP assay}

Chromatin immunoprecipitation and real-time PCR quantification were performed as described (Mukhopadhyay, Deplancke et al., 2008). The rabbit polyclonal COUP-TFIl antibody (Millipore, Cat\# ABE 1426) and rabbit IgG1 antibody (Zymed) were used for immunoprecipitation. After purification of DNA from C3H10T1/2 cells, bound sequences were determined by quantitative real-time PCR (Table 1 for the primer sequences for the ChIP assay).

\section{Western Blot Analysis}

48 hours after transfection, C3H10T1/2 cells were lysed with 1XRIPA buffer containing a protease inhibitor cocktail (Roche Applied Science) for 30 minutes on ice. After centrifugation for 15 minutes, the supernatant was collected, and protein content of the samples was analyzed according to the Bradford method. Proteins were loaded onto SDS-polyacrylamide gels and blotted onto PVDF membrane (Bio-Rad Laboratories). Western blots were performed using antibodies directed against COUP-TFII (abcam, 1: 2000), $\alpha$ SMA (Sigma, 1:4000), and ERK2 (Cell signaling). HRP-conjugated secondary antibodies were purchased from DAKO. Enhanced chemiluminescence was performed according to the manufacturer's instructions. ERK $1 / 2$ protein was used to ensure equivalent loading of protein samples.

\section{Proteomics}

Naïve (WT) and COUP-TFII overexpression (OE) cells were both treated with TGF $\beta 1(10 \mathrm{ng} / \mathrm{ml})$ for 48 hours to induce myofibroblast differentiation. After trypsinization, cells were washed twice in PBS. Cell pellets were frozen in $-80 \mathrm{C}^{\circ}$ for proteomics experiments.

Protein extraction and digest: PBS was removed, followed by addition of SDS lysis buffer (2\% SDS, $150 \mathrm{mM} \mathrm{NaCl}, 50 \mathrm{mM}$ Tris, $\mathrm{pH}$ 8.7) containing protease inhibitors (Complete, Roche). Lysates were homogenized over Qiashredder columns (Qiagen, ref. 79656) and centrifuged at 13,000 rpm for 1 minute at room temperature. Reductive methylation of cysteine residues was performed by adding dithiothreitol (DTT) to a final concentration of $5 \mathrm{mM}$ and heating to $37 \mathrm{C}^{\circ}$ for 1 hour, followed by alkylation with iodoacetamide at a final concentration of $15 \mathrm{mM}$ and incubation at room temperature in the dark for 30 minutes and quenching with DTT. Protein concentration was determined using a Micro BCATM Protein Assay Kit (ThermoFisher, Catalog\# 23235). Detergent was removed by methanol/chloroform protein precipitation as described previously (Wessel \& Flugge, 1984). Lys-C protease digests (Wako, Catalog\# 12902541 ) in $2 \mathrm{M}$ urea $20 \mathrm{mM}$ EPPS, $\mathrm{pH} 8.5$ at $37^{\circ} \mathrm{C}$ for 3 hours were followed by further digestion at $37{ }^{\circ} \mathrm{C}$ for 6 hours with trypsin (Promega, Catalog\# V5113). Missed cleavage rate was determined by LC-MS/MS.

Tandem Mass Tag (TMT) Labeling, Ratio Check and HPLC Fractionation: Equal amounts of protein were removed from each sample and labeled using a TMT11plex Mass Tag Labeling Kit (ThermoFisher, Catalog\# A34808). TMT labeling efficiency and ratio checks were determined by LC-MS3 analysis. Quenched TMT labeling reactions were combined and de- 
salted using a SepPak tC18 Vac RC Cartridge (50 mg, Waters, Catalog\# WAT054960). HPLC fractionation was performed using an Agilent 1200 Series instrument with a flow rate of 600 $\mu \mathrm{l} /$ minute over a period of 75 minutes. Peptides were collected in a 96 -well plate over a $65 \mathrm{~min}-$ gradient of 13-44 \%B with Buffer A comprising 5\% acetonitrile, $10 \mathrm{mM}$ ammonium bicarbonate, $\mathrm{pH} 8$ and Buffer B comprising $90 \%$ acetonitrile, $10 \mathrm{mM}$ ammonium bicarbonate, $\mathrm{pH} 8$. Fractions were then pooled into 24 samples, followed by sample cleanup using the Stage Tip protocol. This protocol uses C18 EmporeTM Extraction Disks (Fisher Scientific, Catalog\# 14-386-2). Samples were dried before re-suspension in MS Loading Buffer (3\% acetonitrile, 5\% FA).

LC-MS: Peptides were separated over a $30 \mathrm{~cm}, 100 \mu \mathrm{m}$ (internal diameter) column using an EASY-nLC 1200 HPLC system. Samples from the HPLC were injected into an Orbitrap Fusion Lumos Tribrid MS (ThermoFisher, Catalog\# FSN02-10000) and measured using a multinotch MS3 method (McAlister, Nusinow et al., 2014, Ting, Rad et al., 2011). MS scans were performed in the Orbitrap over a scan range of $400-1400 \mathrm{~m} / \mathrm{z}$. The top 10 ions with charge states from 2 to 6 were selected for MS/MS. Turbo rate scans were performed in the lon Trap with a collision energy of $35 \%$ and a maximum injection time of $250 \mathrm{~ms}$. TMT quantification was performed using SPS-MS3 in the Orbitrap with a scan range of $100-1000 \mathrm{~m} / \mathrm{z}$ and an HCD collision energy of $55 \%$. Orbitrap resolution was 50,000 (dimensionless units) with a maximum injection time of $300 \mathrm{~ms}$.

Proteomic data analysis: Raw data were converted to mzXML format and peptide ID used Sequest (Eng, McCormack et al., 1994) (version 28 (http://fields.scripps.edu/yates/wp/?page_id=17)) with searches against the Human UniProt database (February 2014). The database search included reversed protein sequences and known contaminants such as human keratins that were excluded for subsequent analyses. Linear discriminant analysis was performed (Elias \& Gygi, 2007) and peptide false discovery rate (FDR) was $<1 \%$ after applying a target-decoy database search strategy. Filtering was performed as described previously (McAlister et al., 2014). Variable modification for oxidized methionine (+15.99 Da) was used during searches. For protein identification and quantification, shared peptides were collapsed into the minimally sufficient number of proteins using rules of parsimony. Peptides with a total TMT value of $>200$ and an isolation specificity of $>0.7$ were included for quantification.

Gene Set Enrichment Analysis (GSEA): We analyzed our proteome datasets from four independent experiments (WT and COUP-TFII-OE, all in duplicates) using the GSEA software developed by Broad Institute (Cambridge, MA) (Subramanian, Tamayo et al., 2005). All the analyses were performed in default setting based on all GO terms (c5.all.v7.0.symbols.gmt).

\section{Real-time cell metabolism assay}

Naïve (WT) and COUP-TFII KO C3H/10T1/2 cells were plated in XF-24 Cell Culture Microplates (Seahorse Bioscience) at a cellular density of 20,000 cells per well, then serum starved for 24 hours and stimulated with or without TGF $\beta 1(10 \mathrm{ng} / \mathrm{ml})$ for 24 hours. Real-time analysis of extracellular acidification rate (ECAR) was analyzed using XF Extracellular Flux Analyzer (Seahorse Bioscience). The cells were incubated in basal media followed by sequential injections with glucose $(10 \mathrm{mM})$, oligomycin $(1 \mu \mathrm{g} / \mathrm{ml})$ and $2-D G(100 \mathrm{mM})$.

\section{Extracellular Lactate Assays}

Extracellular levels of lactate were determined using the lactate assay kit (BioVision, Milpitas, $\mathrm{CA})$ according to the manufacturer's instructions.

\section{Statistical analysis}

Results are expressed as mean \pm SD for three experiments done in triplicate or quadruplicate. Group means were compared by one-way analysis of variance (ANOVA), followed by the Tukey 
post-test using GraphPad Prism (GraphPad software) for multiple comparisons or by student's ttest. A $p$ value $<0.05$ was considered statistically significant.

\section{Acknowledgments}

This work was supported by the National Institutes of Health Grants R37DK039773, R01DKD072381 and UH3TR002155 (to J.V.B.), National Institute of Biomedical Imaging and Bioengineering (NIBIB), Organ Design and Engineering Training Grant 1T32EB016652-01A1 (to L.L). P.G received support from Monahan Foundation, Fondation pour la Recherche Médicale, Groupe Pasteur Mutualité, Société Francophone de Transplantation, Arthur Sachs fellowship, Philippe Foundation, Fulbright Scholarship, ATIP Avenir program. X.X was supported by China Scholarship Council fellowship. We thank Dr. Edy Kim from Brigham women's hospital provided human idiopathic lung disease samples for immunostaining.

Author contributors: LL, PG, XX, ACF, DT, MK and XS performed experiments. LL and JVB designed and analyzed experiments. LL wrote the manuscript.

\section{Conflict of interest}

The authors declare that they have no conflict of interest. 


\section{References}

Akhurst RJ, Hata A (2012) Targeting the TGFbeta signalling pathway in disease. Nat Rev Drug Discov 11: 790-811

Ashraf UM, Sanchez ER, Kumarasamy S (2019) COUP-TFII revisited: Its role in metabolic gene regulation. Steroids 141: 63-69

Avagliano A, Granato G, Ruocco MR, Romano V, Belviso I, Carfora A, Montagnani S, Arcucci A (2018) Metabolic Reprogramming of Cancer Associated Fibroblasts: The Slavery of Stromal Fibroblasts. Biomed Res Int 2018: 6075403

Bao Y, Gu D, Feng W, Sun X, Wang X, Zhang X, Shi Q, Cui G, Yu H, Tang C, Deng A (2014) COUP-TFII regulates metastasis of colorectal adenocarcinoma cells by modulating Snail1. Br J Cancer 111: 933-43

Basak T, Vega-Montoto L, Zimmerman LJ, Tabb DL, Hudson BG, Vanacore RM (2016) Comprehensive Characterization of Glycosylation and Hydroxylation of Basement Membrane Collagen IV by High-Resolution Mass Spectrometry. J Proteome Res 15: 245-58 Bonventre JV, Yang L (2011) Cellular pathophysiology of ischemic acute kidney injury. J Clin Invest 121: 4210-21

Chen X, Qin J, Cheng CM, Tsai MJ, Tsai SY (2012) COUP-TFII is a major regulator of cell cycle and Notch signaling pathways. Mol Endocrinol 26: 1268-77

de Paz-Lugo P, Lupianez JA, Melendez-Hevia E (2018) High glycine concentration increases collagen synthesis by articular chondrocytes in vitro: acute glycine deficiency could be an important cause of osteoarthritis. Amino Acids 50: 1357-1365

Ding H, Jiang L, Xu J, Bai F, Zhou Y, Yuan Q, Luo J, Zen K, Yang J (2017) Inhibiting aerobic glycolysis suppresses renal interstitial fibroblast activation and renal fibrosis. Am J Physiol Renal Physiol 313: F561-F575

Duffield JS (2014) Cellular and molecular mechanisms in kidney fibrosis. J Clin Invest 124: 2299-306

Duffield JS, Lupher M, Thannickal VJ, Wynn TA (2013) Host responses in tissue repair and fibrosis. Annu Rev Pathol 8: 241-76

Elias JE, Gygi SP (2007) Target-decoy search strategy for increased confidence in largescale protein identifications by mass spectrometry. Nat Methods 4: 207-14

Eng JK, McCormack AL, Yates JR (1994) An approach to correlate tandem mass spectral data of peptides with amino acid sequences in a protein database. J Am Soc Mass Spectrom 5: 976-89

Falke LL, Gholizadeh S, Goldschmeding R, Kok RJ, Nguyen TQ (2015) Diverse origins of the myofibroblast-implications for kidney fibrosis. Nat Rev Nephrol 11: 233-44

Fang X, Liu CX, Zeng XR, Huang XM, Chen WL, Wang Y, Ai F (2020) Orphan nuclear receptor COUP-TFII is an oncogenic gene in renal cell carcinoma. Clin Transl Oncol 22: 772-781 Han SH, Wu MY, Nam BY, Park JT, Yoo TH, Kang SW, Park J, Chinga F, Li SY, Susztak K (2017) PGC-1alpha Protects from Notch-Induced Kidney Fibrosis Development. J Am Soc Nephrol 28: 3312-3322

Hou W, Syn WK (2018) Role of Metabolism in Hepatic Stellate Cell Activation and Fibrogenesis. Front Cell Dev Biol 6: 150

Humphreys BD (2018) Mechanisms of Renal Fibrosis. Annu Rev Physiol 80: 309-326 
Humphreys BD, Lin SL, Kobayashi A, Hudson TE, Nowlin BT, Bonventre JV, Valerius MT, McMahon AP, Duffield JS (2010) Fate tracing reveals the pericyte and not epithelial origin of myofibroblasts in kidney fibrosis. Am J Pathol 176: 85-97

Humphreys BD, Valerius MT, Kobayashi A, Mugford JW, Soeung S, Duffield JS, McMahon AP, Bonventre JV (2008) Intrinsic epithelial cells repair the kidney after injury. Cell Stem Cell 2: 284-91

Im MJ, Freshwater MF, Hoopes JE (1976) Enzyme activities in granulation tissue: Energy for collagen synthesis. J Surg Res 20: 121-5

Jha V, Garcia-Garcia G, Iseki K, Li Z, Naicker S, Plattner B, Saran R, Wang AY, Yang CW (2013) Chronic kidney disease: global dimension and perspectives. Lancet 382: 260-72 Kang HM, Ahn SH, Choi P, Ko YA, Han SH, Chinga F, Park AS, Tao J, Sharma K, Pullman J, Bottinger EP, Goldberg IJ, Susztak K (2015) Defective fatty acid oxidation in renal tubular epithelial cells has a key role in kidney fibrosis development. Nat Med 21: 37-46

Klingberg F, Hinz B, White ES (2013) The myofibroblast matrix: implications for tissue repair and fibrosis. J Pathol 229: 298-309

Kobayashi A, Mugford JW, Krautzberger AM, Naiman N, Liao J, McMahon AP (2014) Identification of a multipotent self-renewing stromal progenitor population during mammalian kidney organogenesis. Stem Cell Reports 3: 650-62

Kottmann RM, Kulkarni AA, Smolnycki KA, Lyda E, Dahanayake T, Salibi R, Honnons S, Jones C, Isern NG, Hu JZ, Nathan SD, Grant G, Phipps RP, Sime PJ (2012) Lactic acid is elevated in idiopathic pulmonary fibrosis and induces myofibroblast differentiation via $\mathrm{pH}$ dependent activation of transforming growth factor-beta. Am J Respir Crit Care Med 186: 740-51

Kramann R, Schneider RK, DiRocco DP, Machado F, Fleig S, Bondzie PA, Henderson JM, Ebert BL, Humphreys BD (2015) Perivascular Gli1+ progenitors are key contributors to injury-induced organ fibrosis. Cell Stem Cell 16: 51-66

Lan R, Geng H, Singha PK, Saikumar P, Bottinger EP, Weinberg JM, Venkatachalam MA (2016) Mitochondrial Pathology and Glycolytic Shift during Proximal Tubule Atrophy after Ischemic AKI. J Am Soc Nephrol 27: 3356-3367

Lemos DR, McMurdo M, Karaca G, Wilflingseder J, Leaf IA, Gupta N, Miyoshi T, Susa K, Johnson BG, Soliman K, Wang G, Morizane R, Bonventre JV, Duffield JS (2018) Interleukin1beta Activates a MYC-Dependent Metabolic Switch in Kidney Stromal Cells Necessary for Progressive Tubulointerstitial Fibrosis. J Am Soc Nephrol 29: 1690-1705

Li L, Xie X, Qin J, Jeha GS, Saha PK, Yan J, Haueter CM, Chan L, Tsai SY, Tsai MJ (2009) The nuclear orphan receptor COUP-TFII plays an essential role in adipogenesis, glucose homeostasis, and energy metabolism. Cell Metab 9: 77-87

Li MO, Wan YY, Sanjabi S, Robertson AK, Flavell RA (2006) Transforming growth factorbeta regulation of immune responses. Annu Rev Immunol 24: 99-146 Lin SL, Kisseleva T, Brenner DA, Duffield JS (2008) Pericytes and perivascular fibroblasts are the primary source of collagen-producing cells in obstructive fibrosis of the kidney. Am J Pathol 173: 1617-27

Liu J, Edgington-Giordano F, Dugas C, Abrams A, Katakam P, Satou R, Saifudeen Z (2017) Regulation of Nephron Progenitor Cell Self-Renewal by Intermediary Metabolism. J Am Soc Nephrol 28: 3323-3335 
Madisen L, Zwingman TA, Sunkin SM, Oh SW, Zariwala HA, Gu H, Ng LL, Palmiter RD, Hawrylycz MJ, Jones AR, Lein ES, Zeng H (2010) A robust and high-throughput Cre reporting and characterization system for the whole mouse brain. Nat Neurosci 13: 133-40 McAlister GC, Nusinow DP, Jedrychowski MP, Wuhr M, Huttlin EL, Erickson BK, Rad R, Haas W, Gygi SP (2014) MultiNotch MS3 enables accurate, sensitive, and multiplexed detection of differential expression across cancer cell line proteomes. Anal Chem 86: 7150-8 Meng XM, Huang XR, Xiao J, Chung AC, Qin W, Chen HY, Lan HY (2012) Disruption of Smad4 impairs TGF-beta/Smad3 and Smad7 transcriptional regulation during renal inflammation and fibrosis in vivo and in vitro. Kidney Int 81: 266-79

Morizane R, Lam AQ, Freedman BS, Kishi S, Valerius MT, Bonventre JV (2015) Nephron organoids derived from human pluripotent stem cells model kidney development and injury. Nat Biotechnol 33: 1193-200

Mukhopadhyay A, Deplancke B, Walhout AJ, Tissenbaum HA (2008) Chromatin immunoprecipitation (ChIP) coupled to detection by quantitative real-time PCR to study transcription factor binding to DNA in Caenorhabditis elegans. Nat Protoc 3: 698-709 Nakagawa S, Nishihara K, Miyata H, Shinke H, Tomita E, Kajiwara M, Matsubara T, Iehara N, Igarashi Y, Yamada H, Fukatsu A, Yanagita M, Matsubara K, Masuda S (2015) Molecular Markers of Tubulointerstitial Fibrosis and Tubular Cell Damage in Patients with Chronic Kidney Disease. PLoS One 10: e0136994

Pereira FA, Qiu Y, Tsai MJ, Tsai SY (1995) Chicken ovalbumin upstream promoter transcription factor (COUP-TF): expression during mouse embryogenesis. J Steroid Biochem Mol Biol 53: 503-8

Pereira FA, Qiu Y, Zhou G, Tsai MJ, Tsai SY (1999) The orphan nuclear receptor COUP-TFII is required for angiogenesis and heart development. Genes Dev 13: 1037-49

Picard N, Baum O, Vogetseder A, Kaissling B, Le Hir M (2008) Origin of renal myofibroblasts in the model of unilateral ureter obstruction in the rat. Histochem Cell Biol 130: 141-55 Planchais J, Boutant M, Fauveau V, Qing LD, Sabra-Makke L, Bossard P, Vasseur-Cognet M, Pegorier JP (2015) The role of chicken ovalbumin upstream promoter transcription factor II in the regulation of hepatic fatty acid oxidation and gluconeogenesis in newborn mice. Am J Physiol Endocrinol Metab 308: E868-78

Polvani S, Tarocchi M, Tempesti S, Mello T, Ceni E, Buccoliero F, D'Amico M, Boddi V, Farsi M, Nesi S, Nesi G, Milani S, Galli A (2014) COUP-TFII in pancreatic adenocarcinoma: clinical implication for patient survival and tumor progression. Int J Cancer 134: 1648-58 Principe DR, Doll JA, Bauer J, Jung B, Munshi HG, Bartholin L, Pasche B, Lee C, Grippo PJ (2014) TGF-beta: duality of function between tumor prevention and carcinogenesis. J Natl Cancer Inst 106: djt369

Qin J, Wu SP, Creighton CJ, Dai F, Xie X, Cheng CM, Frolov A, Ayala G, Lin X, Feng XH, Ittmann MM, Tsai SJ, Tsai MJ, Tsai SY (2013) COUP-TFII inhibits TGF-beta-induced growth barrier to promote prostate tumorigenesis. Nature 493: 236-40

Ran FA, Hsu PD, Wright J, Agarwala V, Scott DA, Zhang F (2013) Genome engineering using the CRISPR-Cas9 system. Nat Protoc 8: 2281-2308

Rangarajan S, Kurundkar A, Kurundkar D, Bernard K, Sanders YY, Ding Q, Antony VB, Zhang J, Zmijewski J, Thannickal VJ (2016) Novel Mechanisms for the Antifibrotic Action of Nintedanib. Am J Respir Cell Mol Biol 54: 51-9 Rockey DC, Bell PD, Hill JA (2015) Fibrosis--a common pathway to organ injury and failure. N Engl J Med 372: 1138-49 
Romagnani P, Remuzzi G, Glassock R, Levin A, Jager KJ, Tonelli M, Massy Z, Wanner C, Anders HJ (2017) Chronic kidney disease. Nat Rev Dis Primers 3: 17088

Subramanian A, Tamayo P, Mootha VK, Mukherjee S, Ebert BL, Gillette MA, Paulovich A, Pomeroy SL, Golub TR, Lander ES, Mesirov JP (2005) Gene set enrichment analysis: a knowledge-based approach for interpreting genome-wide expression profiles. Proc Natl Acad Sci U S A 102: 15545-50

Takamoto N, You LR, Moses K, Chiang C, Zimmer WE, Schwartz RJ, DeMayo FJ, Tsai MJ, Tsai SY (2005) COUP-TFII is essential for radial and anteroposterior patterning of the stomach. Development 132: 2179-89

Ting L, Rad R, Gygi SP, Haas W (2011) MS3 eliminates ratio distortion in isobaric multiplexed quantitative proteomics. Nat Methods 8: 937-40

Tomasek JJ, Gabbiani G, Hinz B, Chaponnier C, Brown RA (2002) Myofibroblasts and mechano-regulation of connective tissue remodelling. Nat Rev Mol Cell Biol 3: 349-63 Tran MT, Zsengeller ZK, Berg AH, Khankin EV, Bhasin MK, Kim W, Clish CB, Stillman IE, Karumanchi SA, Rhee EP, Parikh SM (2016) PGC1alpha drives NAD biosynthesis linking oxidative metabolism to renal protection. Nature 531: 528-32

Wessel D, Flugge UI (1984) A method for the quantitative recovery of protein in dilute solution in the presence of detergents and lipids. Anal Biochem 138: 141-3

Wu SP, Kao CY, Wang L, Creighton CJ, Yang J, Donti TR, Harmancey R, Vasquez HG, Graham BH, Bellen HJ, Taegtmeyer H, Chang CP, Tsai MJ, Tsai SY (2015) Increased COUP-TFII expression in adult hearts induces mitochondrial dysfunction resulting in heart failure. Nat Commun 6: 8245

Wynn TA, Ramalingam TR (2012) Mechanisms of fibrosis: therapeutic translation for fibrotic disease. Nat Med 18: 1028-40

Xie N, Tan Z, Banerjee S, Cui H, Ge J, Liu RM, Bernard K, Thannickal VJ, Liu G (2015) Glycolytic Reprogramming in Myofibroblast Differentiation and Lung Fibrosis. Am J Respir Crit Care Med 192: 1462-74

Yang L, Besschetnova TY, Brooks CR, Shah JV, Bonventre JV (2010) Epithelial cell cycle arrest in G2/M mediates kidney fibrosis after injury. Nat Med 16: 535-43, 1p following 143 Zank DC, Bueno M, Mora AL, Rojas M (2018) Idiopathic Pulmonary Fibrosis: Aging, Mitochondrial Dysfunction, and Cellular Bioenergetics. Front Med (Lausanne) 5: 10 Zhao X, Kwan JYY, Yip K, Liu PP, Liu FF (2019) Targeting metabolic dysregulation for fibrosis therapy. Nat Rev Drug Discov 
Table 1. Primers for qRT-PCR and ChIP-qPCR

\begin{tabular}{|l|l|l|}
\hline qRT-PCR & & \\
\hline Target & Primer & Sequence \\
\hline mRPL32 & Forward & GCTGCCATCTGTTTTACGG \\
\hline mRPL32 & Reverse & TGACTGGTGCCTGATGAACT \\
\hline mHexokinase 2 & Forward & CAACTCCGGATGGGACAG \\
\hline mHexokinase 2 & Reverse & CACACGGAAGTTGGTTCTC \\
\hline mGlut1 & Forward & GCT TCT CCA ACT GGA CCT CAA AC \\
\hline mGlut1 & Reverse & ACG AGG AGC ACC GTG AAG ATG A \\
\hline mLDHA & Forward & ACGCAGACAAGGAGCAGTGGAA \\
\hline mLDHA & Reverse & ATGCTCTCAGCCAAGTCTGCCA \\
\hline mPGC1 $\alpha$ & Forward & AGTCCCATACACAACCGCAG \\
\hline mPGC1 $\alpha$ & Reverse & CCCTTGGGGTCATTTGGTGA \\
\hline maSMA & Forward & CTGACAGAGGCACCACTGAA \\
\hline maSMA & Reverse & CATCTCCAGAGTCCAGCACA \\
\hline mCollagen1 & Forward & TGACTGGAAGAGCGGAGAGT \\
\hline mCollagen1 & Reverse & GTTCGGGCTGATGTACCAGT \\
\hline ChIP-qPCR & & \\
\hline Target & Primer & Sequence \\
\hline mPGC1 $\alpha$ & Forward & TTGCCTCCCCTCCTACCTAC \\
\hline mPGC1 $\alpha$ & Reverse & GCATGTTTGCTGGTTGCGTA \\
\hline
\end{tabular}



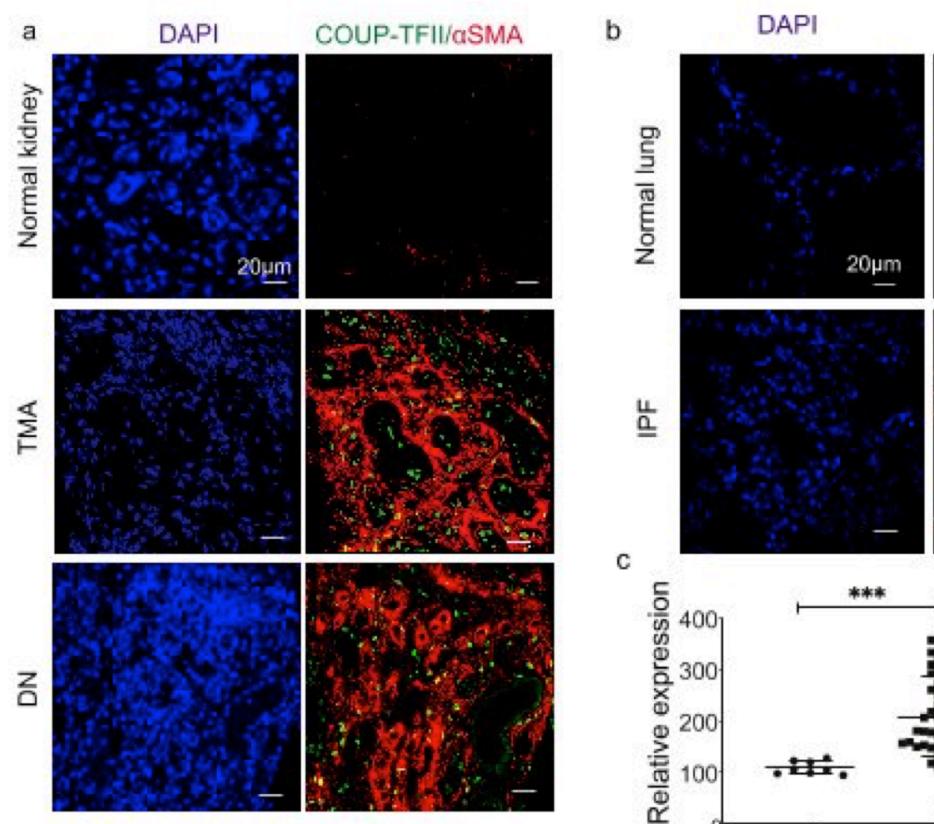

COUP-TFII/aSMA
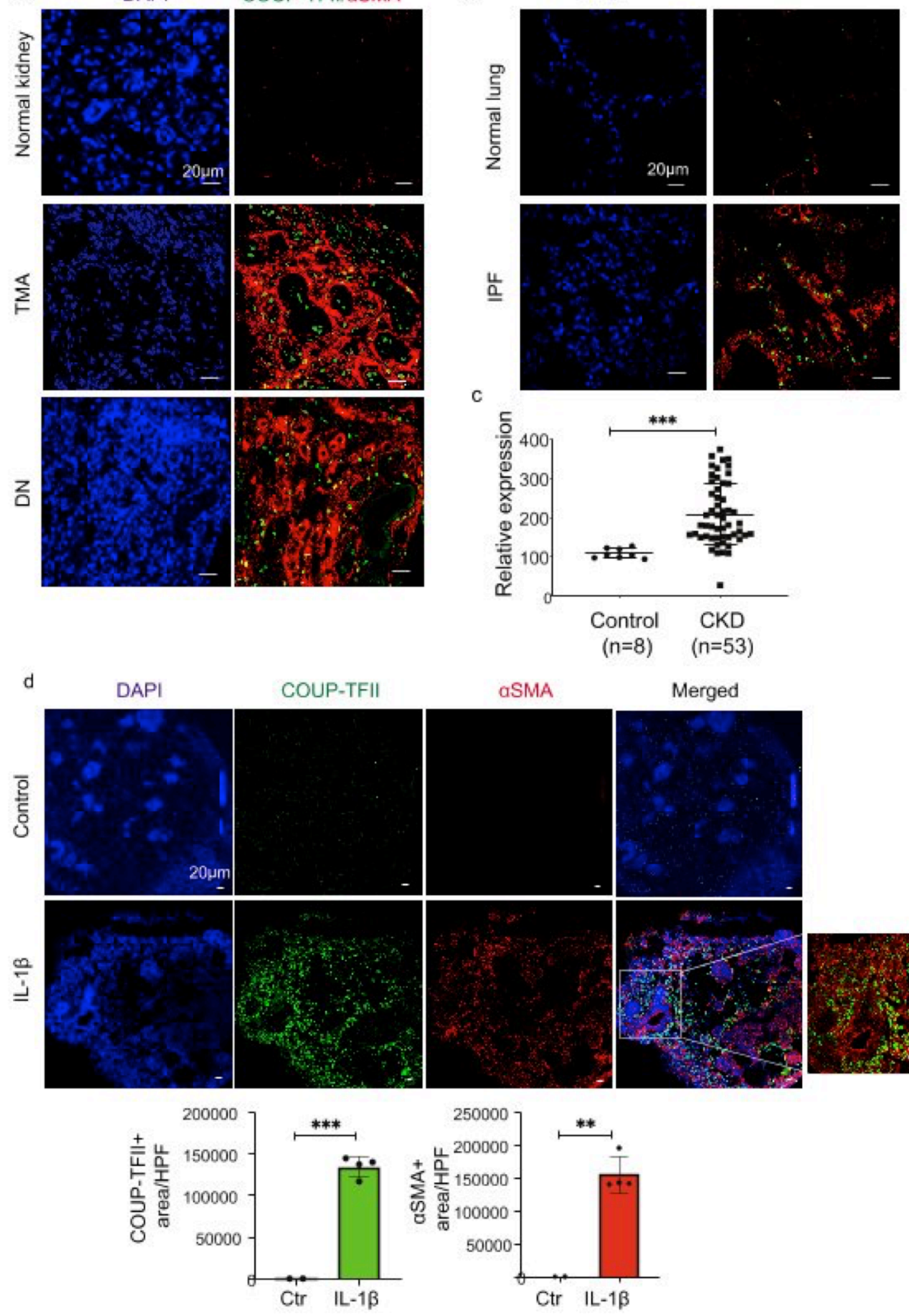

Figure 1. COUP-TFIl expression is increased in human fibrotic kidneys and lungs, and IL$1 \beta$ treated human kidney organoids. COUP-TFII expression is increased in (a) human kidneys from patients with TMA and diabetic nephropathy and (b) in lungs from patients with IPF by immunofluorescence. (c) COUP-TFII mRNA levels in human kidney tissues of control $(n=8)$ and CKD $(n=53)$ subjects from GSE66494. ${ }^{* * *} p<0.001$ by t-test. (d) Human kidney organoids were treated with $\mathrm{IL}-1 \beta(10 \mathrm{ng} / \mathrm{ml})$ for 96 hours. Immunofluorescence reveals significantly increased COUP-TFII and $\alpha$ SMA expression in IL-1 $\beta$ treated organoids, compared to nontreated organoids. Quantification by confocal micrographs in $200 x$ hpf. ${ }^{* * *} p<0.0001$ by t test; mean \pm SD. 
DAPI CD31/COUP-TFII

DAPI PDGFRß/COUP-TFII
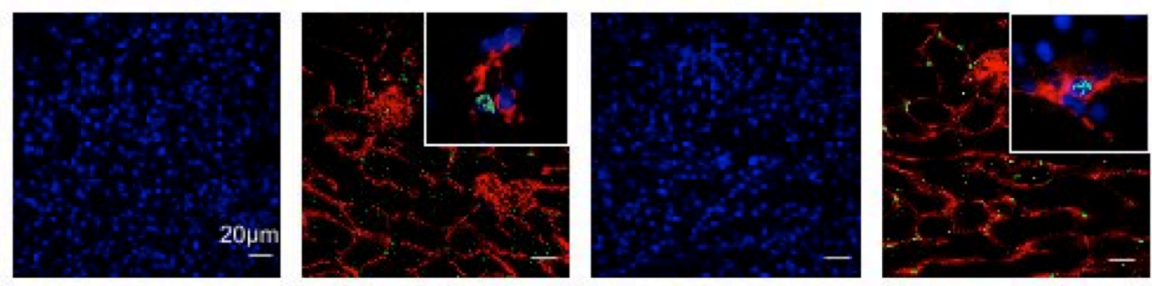

b

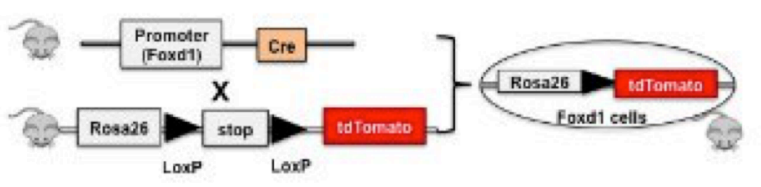

$\mathrm{C}$
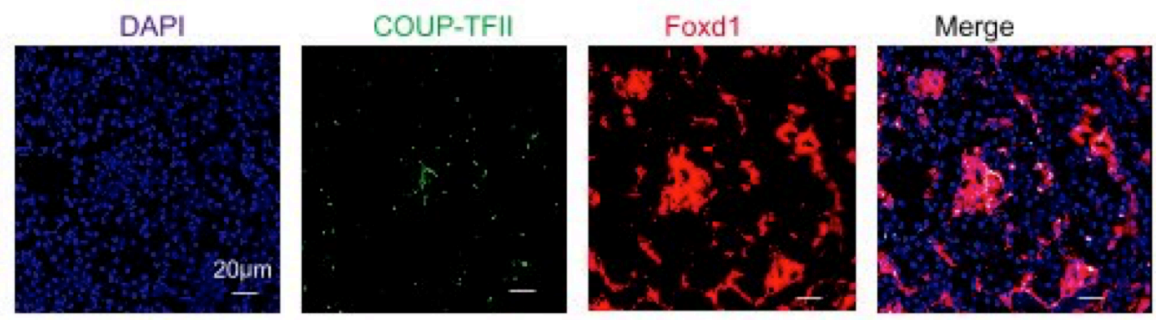

d Human Diabetic Kidney
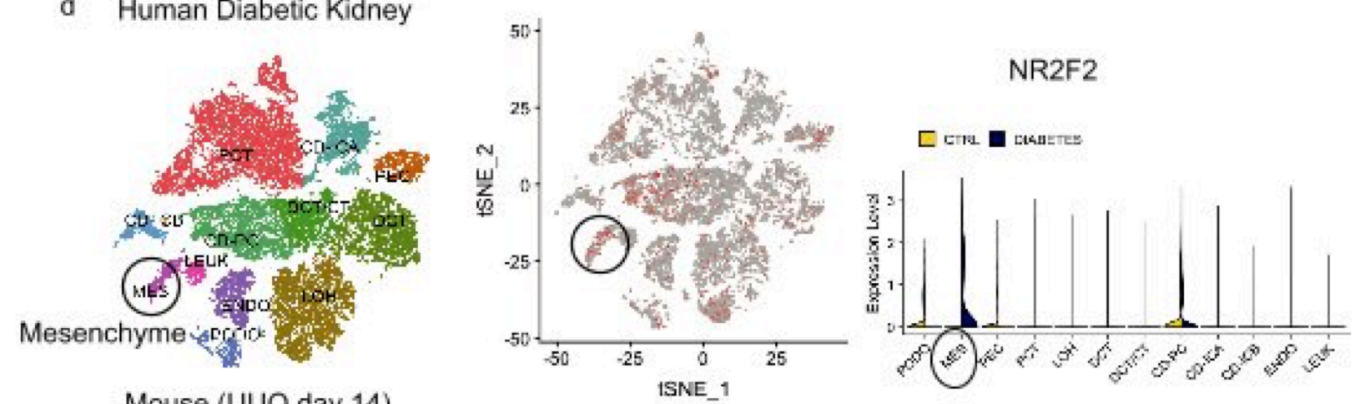

$$
\text { Mouse (UUO day 14) }
$$

w. 1

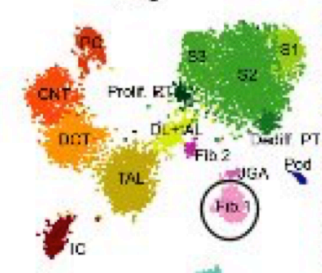

EC.
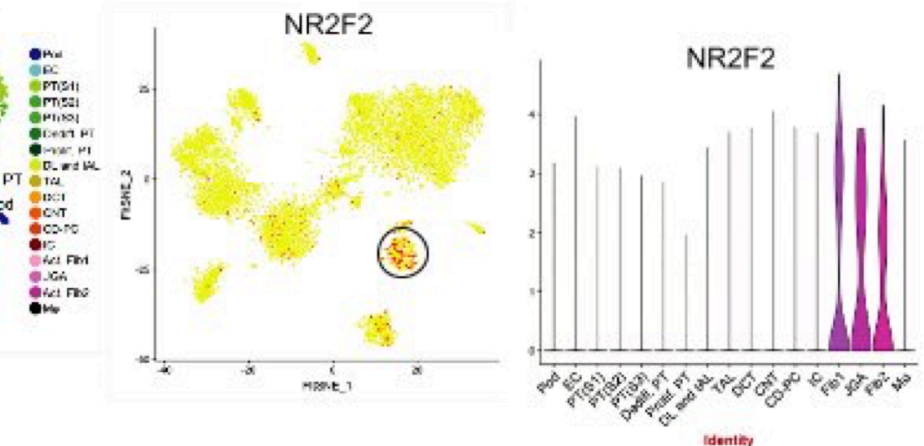

Figure 2. COUP-TFII protein is expressed in pericytes/fibroblasts, both in non-injured and injured mouse kidneys. (a) COUP-TFII+ cells (green) express pericyte/fibroblast marker (PDGFR $\beta$ ) but not the endothelial cell marker (CD31). (b) Strategy of genetically labeling Foxd1derived stromal cells in non-injured mouse kidney. (c) Most COUP-TFII expression cells (green) overlap with tdTomato-labeled Foxd1-derived stromal cells (red) in the non-injured mice kidney. (d) COUP-TFII was enriched most in pericytes/fibroblasts in injured kidney, both in the human (Diabetic kidneys, Fig $2 \mathrm{~d}$ top) and mouse model (UUO day 14, Fig $2 \mathrm{~d}$ bottom) as analyzed from single cell sequencing database (http://humphreyslab.com/SingleCell/). 


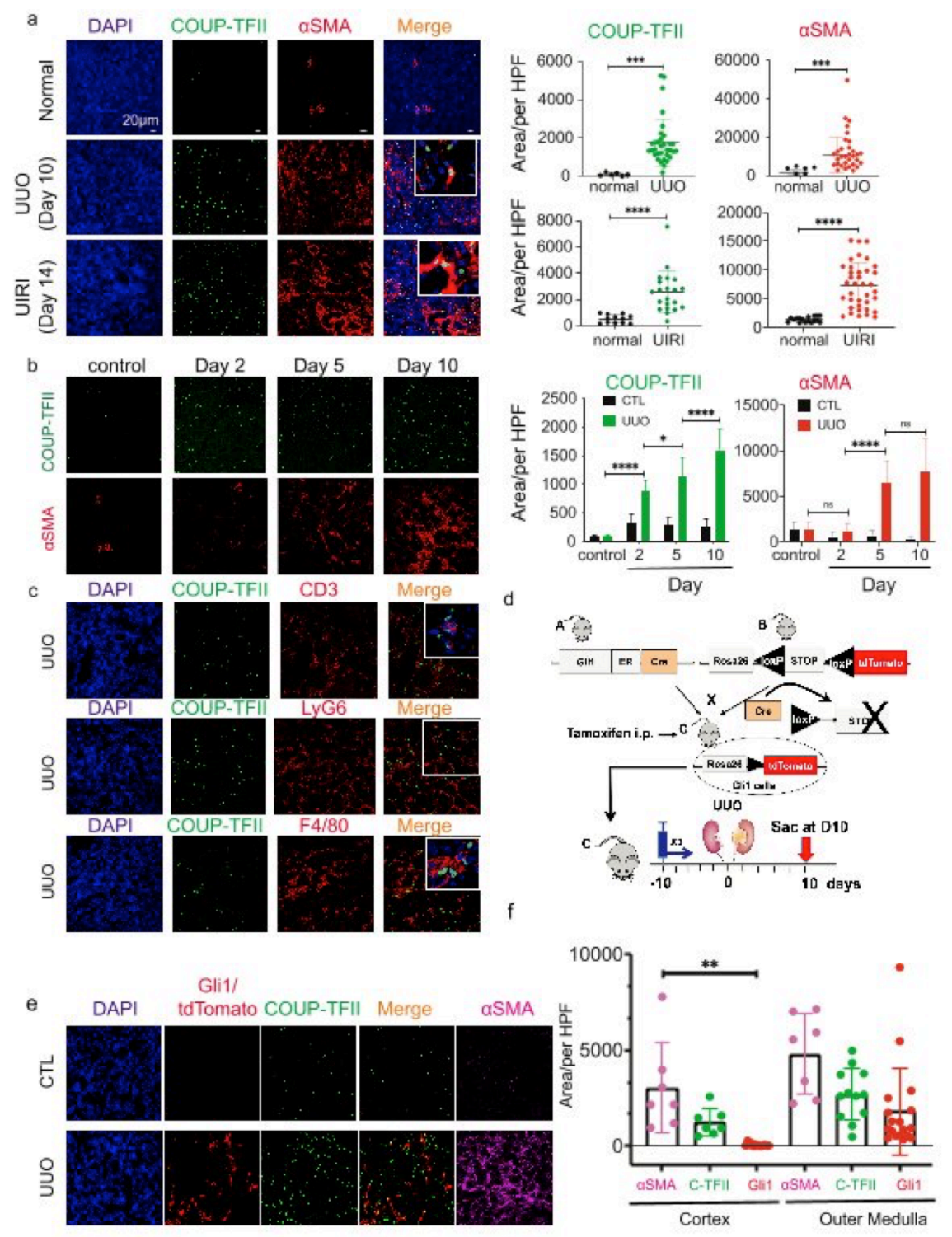

Figure 3. COUP-TFIl expression is increased and co-localized with aSMA+ cells within fibrotic regions in the injured mouse kidneys. (a) COUP-TFII expression is significantly increased and co-localized with aSMA+ cells at day 10 of UUO and day 14 of UIRI in mice ( $n=3$ animals per group). Quantification by confocal micrographs in $200 x$ hpf. ${ }^{* * *} p<0.001$ by $t$ test; mean \pm SD. (b) In a time course of UUO, COUP-TFII expression increased as early as day 2 and preceded the increased expression of $\alpha S M A$ ( $n=3$ animals per time point). Quantification by confocal micrographs in $200 x$ hpf. ${ }^{* * *} p<0.001$ by $\mathrm{t}$ test; mean $\pm \mathrm{SD}$. (c) In the UUO model, COUP-TFII positive cells do not stain with markers of inflammatory cells (T cell marker CD3, neutrophil marker LyG6, and macrophage marker F4/80) ( $n=3$ animals). (d) Using a fate tracing of Gli1+ cells in the UUO model, (e\&f) Gli1-tdTomato+ cells expanded primarily in the outer medullary region. In contrast, COUP-TFII+ cells are distributed both in cortex and medulla. A subset of COUP-TFII+ cells (green) overlap with genetically labeled Gli1+ pericyte/perivascular cells (red) in UUO injury model ( $n=3$ animals per group). CTL: contralateral kidney. Quantification by confocal micrographs in $200 x$ hpf. ${ }^{* * *} p<0.001$ by t test; mean \pm SD. 


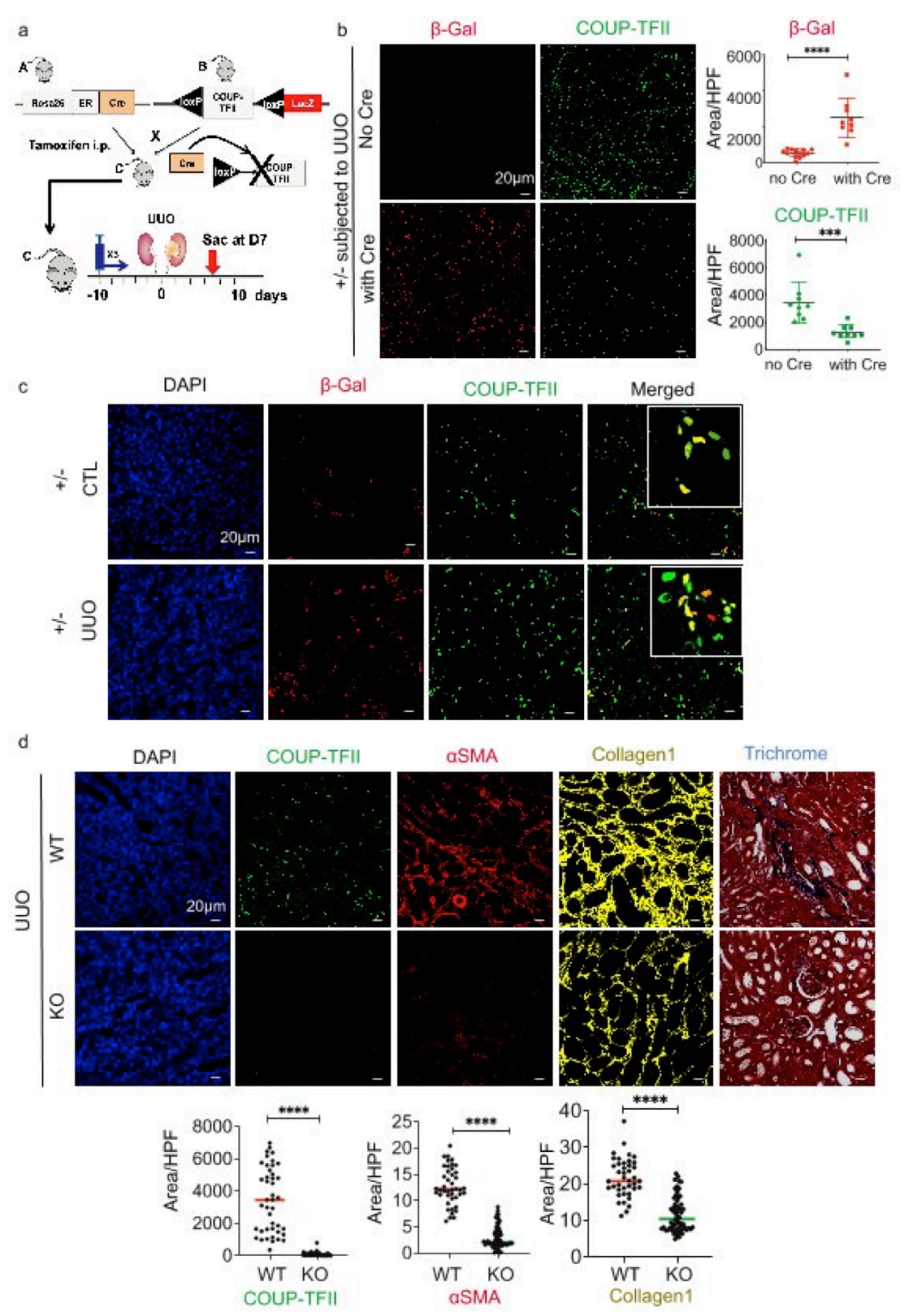

Figure 4. Genetic ablation of COUP-TFII in adult mice attenuates injury-induced kidney fibrosis. (a) Strategy of conditional knockdown of COUP-TFIl in adult mice. A LacZ knock-in allele is inserted into the genomic COUP-TFIl locus after the second LoxP site. (b) Activation of Cre recombinase by Tamoxifen (TAM) results in COUP-TFIl deletion and the expression of LacZ reporter (detected by immunostaining of $\beta$-galactosidase $(\beta-\mathrm{Gal})$ in the UUO mice model $(n=3)$. Quantification by confocal micrographs in $200 x$ hpf. ${ }^{* * *} p<0.001$ by t test; mean \pm SD. (c) Using heterozygous mice ( $\mathrm{F} /+$; Cre/+), $\beta-\mathrm{Gal}+$ cells (red) increased after TAM injection in UUO kidneys, and co-localize with COUP-TFII+ cells (green) $(n=3)$. (d) COUP-TFII+ cells decrease significantly in the UUO kidney in TAM-treated homozygous (F/F;Cre/+) mice (KO group, $n=6$ ) compared to wild-type littermates (WT group, $n=4$ ). Expression of $\alpha$ SMA (red) and collagen 1 (yellow) are also markedly reduced. Masson Trichrome staining shows less kidney fibrosis in KO compared to WT group. Quantification by confocal micrographs in 200x hpf. There are 8-10 images taken and quantified for each animal (represented by each dot). ${ }^{* *} p<0.001$ by $t$ test, mean \pm SD. 
a

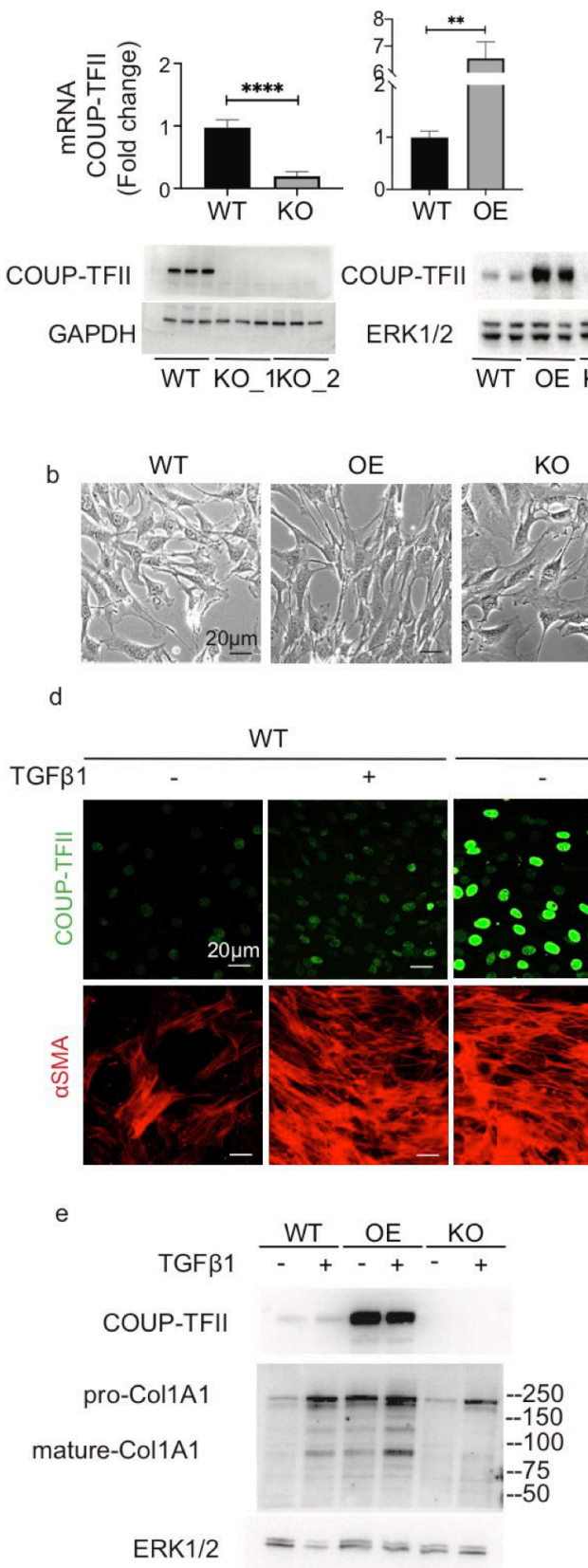

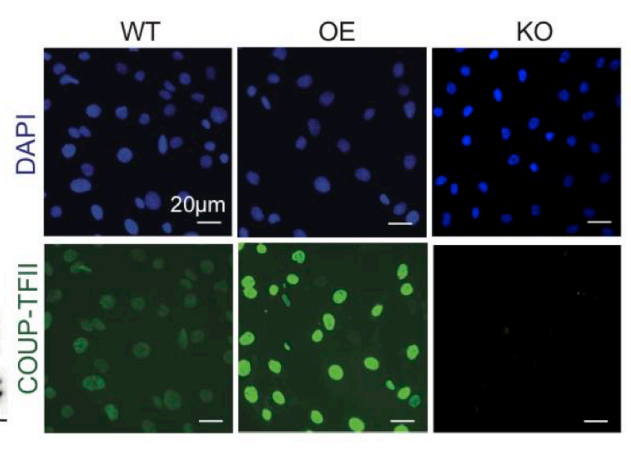

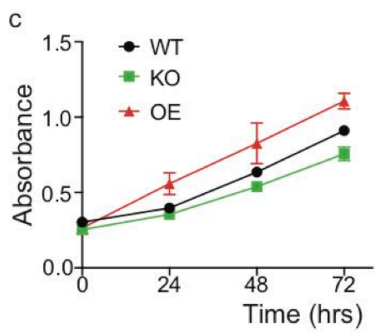

OE KO
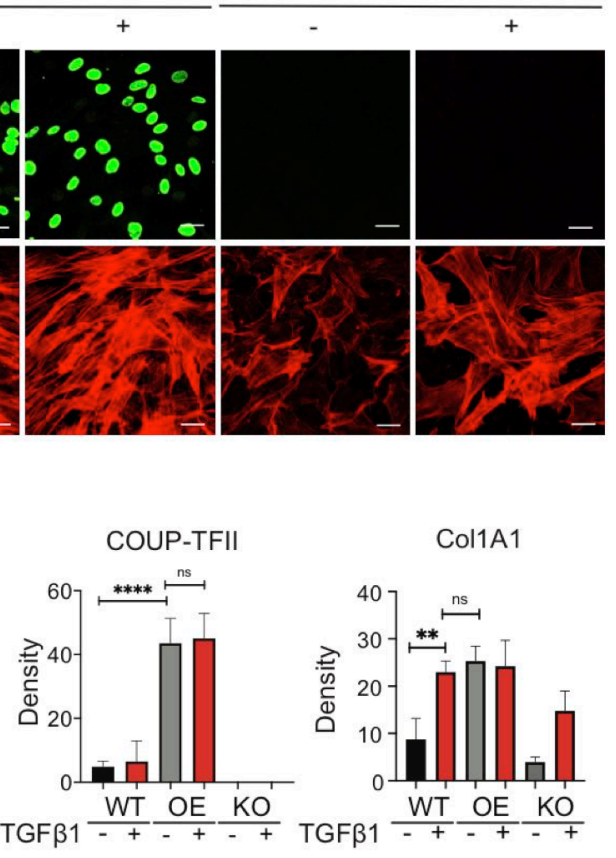

Figure 5. Cellular knockout of COUP-TFII in vitro in $\mathrm{C} 3 \mathrm{H} / 10 \mathrm{~T} 1 / 2$ cells decreases cell proliferation and suppresses TGF $\beta 1$-induced $\alpha$ SMA and collagen 1 expression. In contrast, overexpression of COUP-TFII alone increases collagen1 production. (a)

Verification of COUP-TFII loss- and gain-of-function cell lines generated by CRISPR-Cas9 and an inducible lentiviral construct in pericyte-like cell line C3H/10T1/2 in vitro $(n=6)$. (b\&c) COUPTFII KO had no effect on cell viability, although decreased proliferation rate compared to naïve C3H/10T1/2 cells (WT) $(n=6),{ }^{* * * *} p<0.0001$ by two-way ANOVA. (d) COUP-TFII-OE cells, in the absence of TGF $\beta 1$ stimulation, displayed an elongated fibroblast shape, similar to WT cells treated with TGF $\beta 1$. (e) Overexpression of COUP-TFII alone without TGF $\beta 1$ induces collagen1 production $(n=3) .{ }^{* * * *} p<0.0001$ by one-way ANOVA, mean \pm SD. 


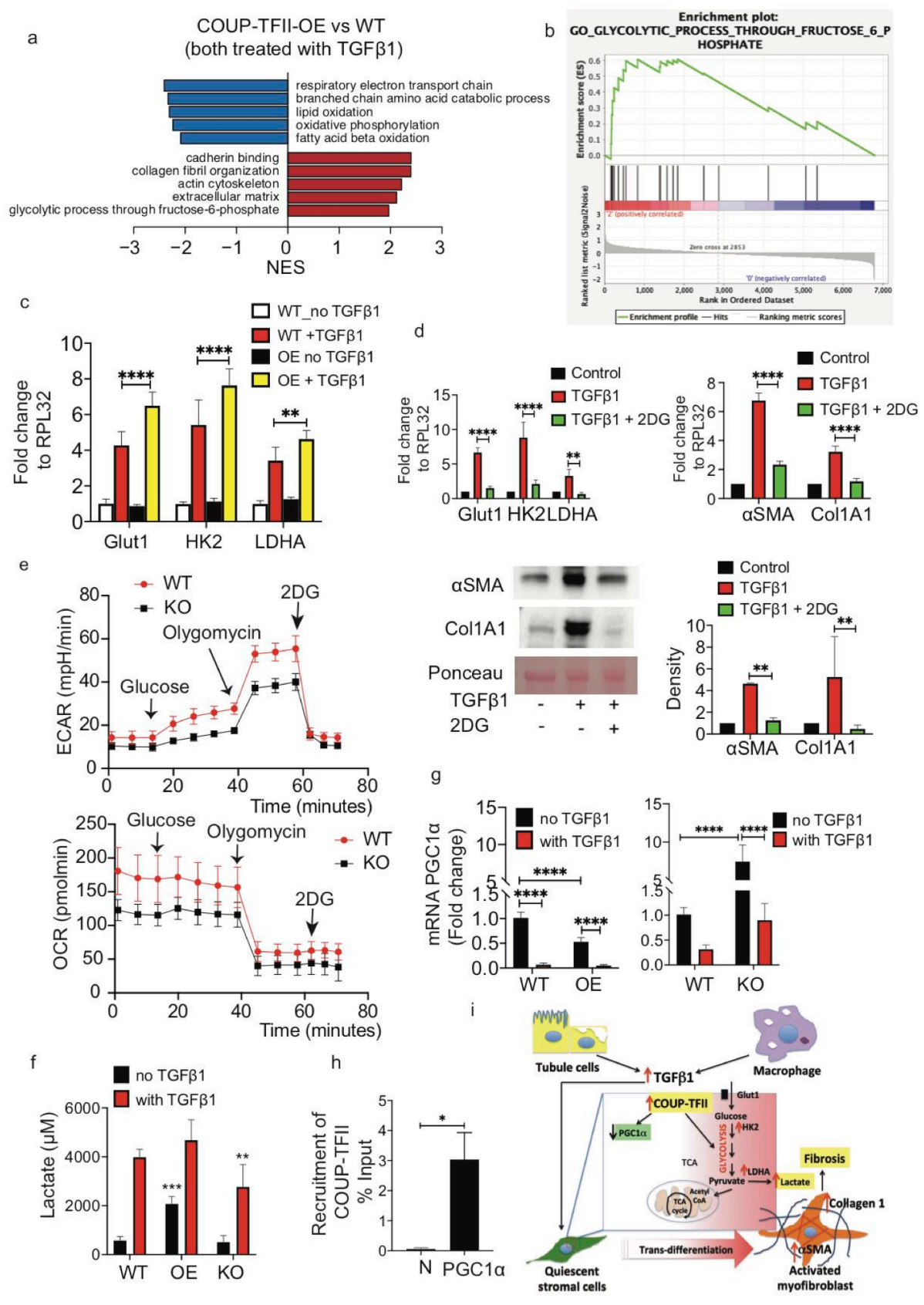

Figure 6. COUP-TFII regulates myofibroblast differentiation through metabolic reprogramming in vitro. (a\&b) Non-biased proteomics of TGF $\beta 1$-treated naïve (WT) and COUP-TFII overexpressing (COUP-TFII-OE) cells reveals a metabolic shift from fatty acid oxidation to glycolysis. Top pathways ranked by NES (gene set enrichment with FDR < 0.05) altered in COUP-TFII-OE compared to naïve C3H10T1/2 cells $(n=2)$. (c) qRT-PCR of glycolysis genes for WT and COUP-TFII-OE cells treated without or with TGF $1 \quad(10 \mathrm{ng} / \mathrm{ml})(\mathrm{n}=6)$. ${ }^{* *} p<0.01,{ }^{* * * *} p<0.0001$ by two-way ANOVA. (d) 2-deoxyglucose (2-DG) treatment inhibits TGF $\beta 1$-induced glycolysis (left) and fibrosis (right) in COUP-TFII-OE cells by qRT-PCR $(n=6)$. Collagen 1 protein is reduced by $2-D G$ confirmed by western blot $(n=3)$. ${ }^{* * *} p<0.0001$ by oneway ANOVA. (e) WT and COUP-TFII knockout (COUP-TFII-KO) cells were seeded in Seahorse 
XF-24 cell culture microplates. The cells were rendered quiescent in 1\% BSA DMEM overnight and then treated with $10 \mathrm{ng} / \mathrm{ml}$ TGF $\beta 1$ for 24 hours, followed by sequential treatments with glucose $(10 \mathrm{mM})$, oligomycin $(1 \mu \mathrm{g} / \mathrm{ml})$ and 2-DG $(100 \mathrm{mM})$. Real-time extracellular acidification rate $(E C A R)$ was recorded $(n=10)$. When compared with WT, COUP-TFII-KO cells significantly decreased ECAR induced by TGF $\beta 1$. Similar difference were seen after oligomycin injection, which indicated the glycolytic capacity. ${ }^{* * *} \mathrm{p}<0.0001$ by t test. Interestingly, KO cells displayed a lower oxygen consumption rate (OCR), a measure of mitochondrial respiratory activity, in response to TGF $\beta 1$ compared with WT cells. (f) TGF $\beta 1$ significantly increased more lactate production by WT $\mathrm{C} 3 \mathrm{H} / 10 \mathrm{~T} 1 / 2$ cells when compared to COUP-TFII-KO cells treated with TGF $\beta 1$. In contrast, overexpression of COUP-TFII alone (without TGF $\beta 1$ ) significantly increased lactate $(n=3) .{ }^{* *} p<0.01,{ }^{* * *} p<0.001$ by one-way ANOVA, mean \pm SD. (g) Overexpression of COUP-TFII inhibited, and knockout of COUP-TFII increased, PGC1 a mRNA $(n=6) .{ }^{* * *} p<0.001$ by one-way ANOVA, mean \pm SD. (h) Chip-qPCR analysis on C3H10T1/2 cells in vitro revealed binding of COUP-TFII on the promoter of PGC1a $(n=2)$. ${ }^{*} p<0.05$, mean \pm SD. (i) Schematic model of the role of COUP-TFII in metabolic reprogramming during myofibroblast differentiation and fibrosis formation after injury. 